\title{
Static Aeroelastic Model Validation of Membrane Micro Air Vehicle Wings
}

\author{
Bret Stanford ${ }^{1}$ \\ Department of Mechanical and Aerospace Engineering, University of Florida, Gainesville, FL 32611-6250 \\ Roberto Albertani ${ }^{2}$ \\ Research and Education Engineering Facility, University of Florida, Shalimar, FL 32579-6250 \\ Dragos Viieru ${ }^{3}$ and Wei Shyy ${ }^{4}$ \\ Department of Aerospace Engineering, University of Michigan, Ann Arbor, Michigan, 48109-2140 \\ and \\ Peter Ifju ${ }^{5}$ \\ Department of Mechanical and Aerospace Engineering, University of Florida, Gainesville, FL 32611-6250
}

\begin{abstract}
A low aspect ratio, low Reynolds number membrane wing has been identified as a viable platform for micro air vehicle applications. Desirable flying qualities include high lift and larger stability margins. Several challenges are associated with the numerical modeling of such a wing, including highly three-dimensional flows, separation bubbles, and nonlinear membrane behavior. A thorough model validation and system identification effort is therefore required. A novel experimental setup integrates a wind tunnel with a visual image correlation system, for simultaneous measurement of wing displacements, strains, and aerodynamic loads. These three metrics are used for a direct comparison of numerical and experimental data for both pre- and post-stall angles of attack. Suitable correspondence is demonstrated for moderate angles of attack; methods for increasing the model fidelity can be made for angles with poor predictive capability. Computed flow structures reveal further information concerning the aeroelastic behavior of membrane wings.
\end{abstract}

\section{Nomenclature}

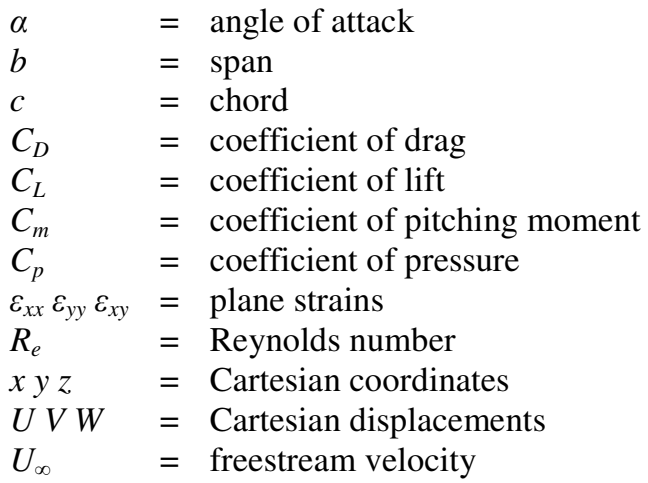

\footnotetext{
${ }^{1}$ Research Assistant, MAE, University of Florida, Gainesville, FL 32611.

${ }^{2}$ Visiting Professor, REEF, University of Florida, Shalimar, FL 32579.

${ }^{3}$ Postdoctoral Research Fellow, AE, University of Michigan, Ann Arbor, MI 48109.

${ }_{5}^{4}$ Clarence L. "Kelly" Johnson Collegiate Professor and Chair, AE, University of Michigan, Ann Arbor, MI 48109.

${ }^{5}$ Professor, MAE, University of Florida, Gainesville, FL 32611.
} 


\section{Introduction}

A hallmark of many practical micro air vehicle designs is a thin, cambered, low aspect ratio wing (typically on the order of one). This approach maximizes the wing area for a given size constraint (150 mm, by definition), thus improving the flight duration for a given payload (which will include motors, actuation servos, and receivers, and may include video cameras or autopilot systems). Further desire to minimize the size of a MAV has lead to the elimination of horizontal stabilizers, providing a "flying wing". Such a wing requires a reflex airfoil for stability, wherein the re-curve present towards the trailing edge can help offset the longitudinal pitching moment of the remainder of the wing. High-velocity electric motors power small propellers for vehicle propulsion. A micro air vehicle designed, fabricated, and flight-tested at the University of Florida can be seen in Figure 1.

Researchers at the University of Florida have identified a series of thin, flexible, lightweight materials well suited to the fabrication of micro air vehicles ${ }^{1}$ : composite laminates and thin extensible latex rubber membranes. The leading edge of the wing seen in Figure 1 is constructed from a bi-directional plain weave carbon fiber laminate, as is the thin curved strip (perimeter) that outlines the planform. The remainder of the wing (outside of the windowed hatch at the root) is composed of the rubber membrane skin, which is sealed to the carbon fiber with spray adhesive. This combination of carbon fiber skeleton and membrane skin affords a wing that is lightweight enough to fly in the appropriate flight regime (MAV speeds may reach $13 \mathrm{~m} / \mathrm{s}$, which equates to a relatively low Reynolds number of $10^{5}$ ), but strong enough to sustain the resulting lift forces. Further benefits include durability, ease of fabrication, and most importantly, passive shape adaptation.

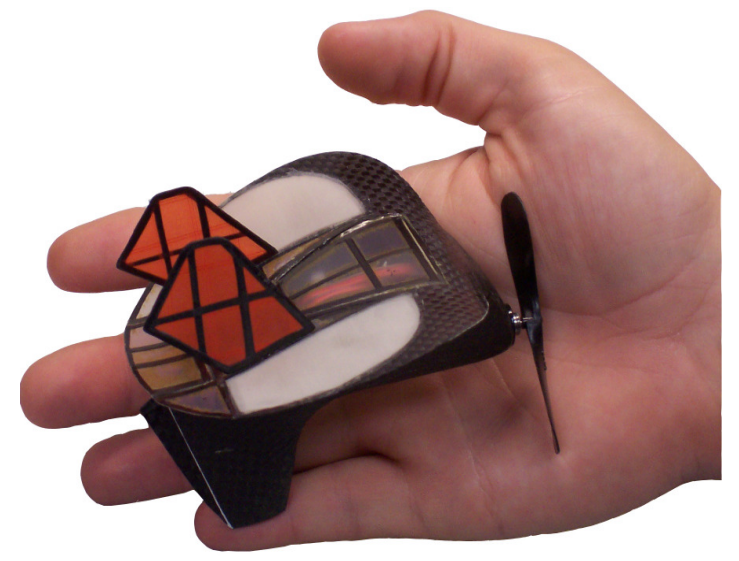

Figure 1. University of Florida micro air vehicle.

The MAV wing's aerodynamic performance is significantly affected by the adaptive inflation of the latex membrane skin. Desirable aeroelastic effects include higher lift (due to the adaptive camber) and improved longitudinal static stability (as the maximum camber shifts towards the trailing edge); the resulting non-optimal aerodynamic wing shape will incur a drag penalty, however. This work details the development of a static aeroelastic model of the adaptively inflating membrane MAV wing. Several challenges are associated with such a modeling effort: the low aspect ratio wing forces a highly three-dimensional flow, the low Reynolds number implies strong viscous effects such as flow separation/reattachment, the mechanics of the membrane inflation is inherently nonlinear, etc. As such, a series of experimental techniques are used for model validation and system identification purposes. Finally, both numerical and experimental data (in terms of wing displacement, strain, and pressure fields and longitudinal aerodynamic coefficients) will be used to facilitate a greater understanding of the fluid-structure interaction of a membrane micro air vehicle wing.

Numerical modeling of membrane wings is well represented in the literature. Jackson ${ }^{2}$ derived and solved an equation for an extensible two-dimensional sailwing (fixed at the leading and trailing edge) in inviscid flow at an arbitrary angle of attack. Later work by Smith and Shyy ${ }^{3}$ studied the same problem with a Reynolds-averaged Navier-Stokes solver. The data indicated that potential flow solvers can provide meaningful insight into the physics of the problem only for small angles of attack and for small membrane excess ratios; otherwise viscous effects within the surrounding flow dominate. Early work on the aeroelasticity of a three-dimensional membrane wing is conducted by Boudreault ${ }^{4}$. The author used an inviscid vortex lattice method (VLM), and described the wing section shapes as cubic polynomials: the aeroelastic solution was given in terms of the excess length within the fabric. Jackson and Christie ${ }^{5}$ used a nonlinear membrane finite element coupled to a VLM to study a thin triangular sail. They found that fixing the trailing edge for adaptive camber provided the highest lift. Sugimoto ${ }^{6}$ found 
solutions to the static aeroelasticity of a fully restrained (about the leading edge, trailing edge, and wing tips) elastic circular wing, and was able to obtain good correlation to experimental results. Lian et al. ${ }^{7}$ computed the aeroelasticity of a membrane MAV with three battens imbedded within each wing. A time dependent Navier-Stokes flow solver was coupled with a nonlinear hyperelastic membrane model. The data indicated that the membrane wing experiences a self-excited vibration on the order of $100 \mathrm{~Hz}$, with a maximum wing velocity about $2 \%$ of the freestream. Camber, incidence, and wing deformation at various span locations are documented as a function of angle of attack.

Early wind tunnel work by Fink ${ }^{8}$ tested a sail wing with a membrane fabric skin. Prior to stall, the deformation profile of the wing was fairly smooth, but rippling developed as the wing stalled. At low angles of attack, the lift curve is reported to be unusually steep, due to the adaptive camber. Fink notes that the pretension within the wing has a significant effect upon the aerodynamic characteristics. Greenhalgh and Curtiss ${ }^{9}$ conducted similar tests with varying planform shapes: triangular, elliptical, and parabolic. They found that, for the range of tested flight conditions, only the parabolic planform shape could support the loading without the aid of a trailing edge support member, and exhibited superior aerodynamic performance. Galvao et al. ${ }^{10}$ conduct wind tunnel testing on a latex membrane sheet stretched between two rigid posts. Photogrammetry was used to measure the membrane displacements. As above, the steep lift slopes were reported, as well as smoother stall behavior. The latter is due to a de-cambering of the wing, as the pressure over the upper surface increased due to imminent flow separation. Fleming et al. ${ }^{11}$ discuss the use of projection moiré interferometry for measuring the deformation of a low aspect ratio membrane MAV wing in a wind tunnel. DeLuca et al. ${ }^{12}$ reports the benevolent stall behavior of flexible MAV wings, citing a maximum L/D ratio $20 \%$ to $30 \%$ larger than that measured from a rigid wing. MAV's with membrane wings displayed static stability in all three principle control axes, whereas the vehicle with a rigid wing was not definitely stable in any axis.

The remainder of this work is organized as follows. A complete description of all the mechanisms used to provide information pertaining to membrane wing aeroelasticity (closed loop wind tunnel, strain gage sting balance, visual image correlation, finite element analysis, computational fluid dynamics, aeroelastic coupling) will be given, as well as a discussion of the particular MAV prototype used for testing. System identification for the relevant structural parameters will be described, followed by model validation via wing deformation and longitudinal aerodynamic coefficients. Once a suitable level of confidence in the aeroelastic model is obtained, the computed flow structures are discussed in detail.

\section{Experimental Techniques}

\section{A. Closed Loop Wind Tunnel}

The test facility used for the entirety of this work is an Engineering Laboratory Design (ELD) 407B closed loop wind tunnel, with the flow loop arranged in a horizontal configuration. The test section has an inner dimension of $0.84 \mathrm{~m}$ on each side, and is $2.44 \mathrm{~m}$ deep. The velocity range is between 2 and $45 \mathrm{~m} / \mathrm{s}$ : the maximum $\mathrm{Re}$ is 2.7 million. Centerline turbulence levels have been measured on the order of $0.2 \%$. Optical access is available on the sidewalls and the ceiling. A Heise model PM differential pressure transducer is attached to a pitot-static tube located at the center of the section's entrance: the system is capable of measuring wind speeds up to $45 \mathrm{~m} / \mathrm{s}$. A fourwire RTD mounted to the wall of the test section measures the airflow temperature.

\section{B. Strain Gage Sting Balance}

An Aerolab 01-15 6-component strain gage sting balance is used to measure the aerodynamic forces and moments of the wind tunnel models. Each of the six channels is in a full Wheatstone-bridge configuration, with 5 channels dedicated to forces, and 1 to a moment. The forces generated during MAV flight may not be more than a few percent of the maximum measurable load of each channel. Electrical signals are on the order of microvolts, which introduces a challenging data processing and signal conditioning problem. The balance's drag signal, for example, is calibrated down to $0.01 \mathrm{~N}$, which is about $40 \%$ of the minimum theoretical drag expected from a MAV. Data acquisition is done with a NI SCXI 15208 channel programmable strain gage module with full bridge configuration, 2.5 excitation volts, and a gain of 1000. A NI 6052 DAQ PAD firewire provides A/D conversion, multiplexing, and the PC connection.

For a given flight condition, the output signals from the six components are sampled at $1000 \mathrm{~Hz}$ for 2 seconds. The average of this data is sent to one module for the calculation of the relevant aerodynamic coefficients $\left(C_{L}, C_{D}\right.$, $\mathrm{C}_{\mathrm{m}}$, etc), and the standard deviation of the data is stored for an uncertainty analysis. Corrections are applied to the aerodynamic coefficients to account for blockage (solid, wake and streamline curvature) and flexibility effects. The sting balance is mounted to a custom-fabricated aluminum model arm within the section. The arm extends through a 
hole in the section wall, and is then attached to a gearbox and a brushless servomotor system for pitching control (rates on the order of $1 \% \mathrm{~s}$ ). Tunnel speed, model inclination, and force/moment measurements are set/acquired using a dedicated PC and in-house software. A schematic of the wind tunnel setup can be seen in Figure 2.

\section{Visual Image Correlation}

Visual Image Correlation (VIC) is a non-contacting full-field measurement technique originally developed by researchers at the University of South Carolina ${ }^{13,14}$. The underlying principle is to calculate the displacement field of a test specimen by tracking the deformation of a random speckling pattern applied to the surface. Two precalibrated cameras digitally acquire this pattern before and after loading, using stereo-triangulation techniques. The VIC system then tries to find a region (in the image of the deformed specimen) that maximizes a normalized crosscorrelation function corresponding to a small subset of the reference image (taken when no load is applied to the structure). The image space is iteratively swept by the parameters of the cross-correlation function to transform the coordinates of the original reference frame to coordinates within the deformed image. As it is unlikely that the deformed coordinates will directly fall onto the sampling grid of the reference image, accurate grey-value interpolation schemes are implemented to achieve optimal sub-pixel accuracy without bias.

In order to capture the three-dimensional features and deformation of a wind tunnel model, twin synchronized cameras, each looking from a different viewing angle, are installed above the wind tunnel ceiling (seen in Figure 2). As the cameras remain stationary throughout the experiment, a mounting bracket straddles the tunnel to prevent the transmission of vibration. Optical access into the test section is through a glass ceiling. Two continuous $250 \mathrm{~W}$ lamps illuminate the model, enabling the use of exposure times of 5 to $10 \mathrm{~ms}$. The energy emitted from the lights, a potential hazard for the specimen (particularly the thin membrane skin, whose elastic properties are known to degrade in adverse conditions), was not a concern due to the cooling effect of the wind tunnel flow.

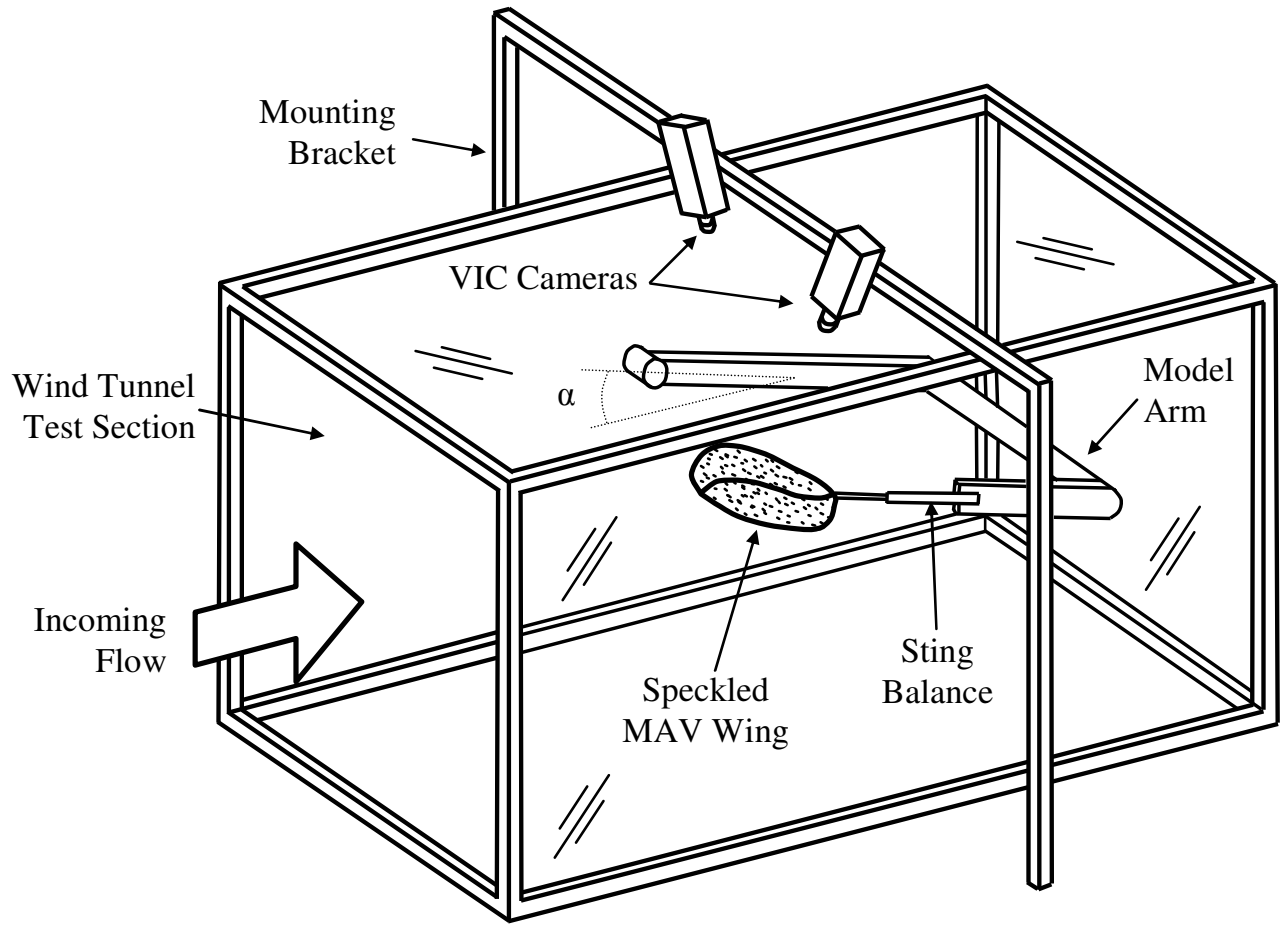

Figure 2. Schematic of wind tunnel setup.

The twin cameras are connected with a PC via an IEEE 1394 firewire cable, and a specialized unit is used to synchronize the camera triggers for instantaneous shots. A standard acquisition board installed in the computer carries out digitalization of the images, and the image processing is carried out by custom software, provided by Correlated Solutions, Inc. Typical data results obtained from the VIC system consist of geometry of the surface in discrete coordinates $(\mathrm{x}, \mathrm{y}, \mathrm{z})$ and the corresponding displacements $(\mathrm{U}, \mathrm{V}, \mathrm{W})$. The VIC system places a grid point every $\mathrm{N}$ pixels, where $\mathrm{N}$ is user defined. A final post-processing option involves calculating the in-plane strains $\left(\varepsilon_{\mathrm{xx}}\right.$, $\varepsilon_{\mathrm{y} y}$, and $\left.\varepsilon_{\mathrm{xy}}\right)$. This is done by mapping the displacement field onto an unstructured triangular mesh, and conducting the appropriate numerical differentiation (the complete definition of finite strains is used). 
The general procedural steps used in this work are:

1) Take a picture of the wind tunnel model at the set angle of attack, with the wind off.

2) Start the wind tunnel, and wait for stable conditions.

3) Take a picture of the deformed wing, and record the aerodynamic loads.

4) Stop the wind tunnel, move the model to the next angle, and repeat.

Each pair of images is then sent to the VIC system for processing. The acquired displacement field is composed of both the elastic deformation of the wing and the rigid body motions inherent within the wind tunnel setup. These motions are thought to primarily originate from the flexibility of the sting balance, and must be filtered out.

\section{Specimen Selection and Preparation}

Only the wing (125 mm wingspan, $104 \mathrm{~mm}$ root chord) of the MAV seen in Figure 1 is considered in this work. The leading edge, perimeter (curved strip that surrounds the latex membrane wing skin) and inboard portion of the wing are constructed from 6 layers of plain weave carbon fiber plies, each in the $\left[ \pm 45^{\circ}\right]$ orientation. Out of weight considerations, this is a thicker laminate than would be implemented for practical MAV design. The excessive stiffness ensures that the majority of strain energy due to aerodynamic loading is within the membrane (rather than bending/twisting of the laminate), thus simplifying the numerical modeling effort. After the curing cycle, the carbon fiber wing skeleton is painted a light color. A random speckling pattern (black spray paint droplets) is then applied to a sheet of latex rubber. The sheet is stretched about a frame, pinned along the boundary to hold the tension, and then sealed to the laminate skeleton by a spray glue adhesive. After the glue has dried, the excess latex is trimmed away. VIC can be used to measure the resulting pre-strain in the MAV's membrane wing, by taking images before and after the sheet is adhered to the skeleton.

\section{Numerical Techniques}

\section{A. Computational Fluid Dynamics}

The three-dimensional, incompressible Navier-Stokes equations written in curvilinear coordinates are solved for the steady, laminar flow over a MAV wing with a span of $125 \mathrm{~mm}$. The fuselage, stabilizers, and propeller are not taken into account. The computational domain can be seen in Figure 3, with the MAV wing (red) enclosed within. Inlet and outlet boundaries are marked by the flow vectors: velocity is specified at the inlet, while a zero pressure condition is enforced at the outlet. The configuration shown in Figure 3 is for simulations at a model inclination of $0^{\circ}$. For non-zero angles, the lower and upper surfaces will also see a mass flux. The side walls are modeled as slip walls, and thus no boundary layer forms. The dimensions of the computational domain are given in terms of the root chord, and are placed far enough away from the MAV surface so as not to significantly affect the aerodynamics. As no flow is expected to cross the root chord of the wing (no propeller is modeled), symmetry is exploited by modeling only half of the computational domain (the plane of symmetry is also modeled as a slip wall). A detailed view of the resulting structured mesh is given in Figure 3. 210,000 nodes fill half of the computational domain, with 1300 nodes on the wing surface. The Navier-Stokes equations are solved on this mesh using a finite volume formulation. A second order central difference operator is used for pressure and diffusive terms, while a second order upwind scheme handles all convective terms ${ }^{15}$.
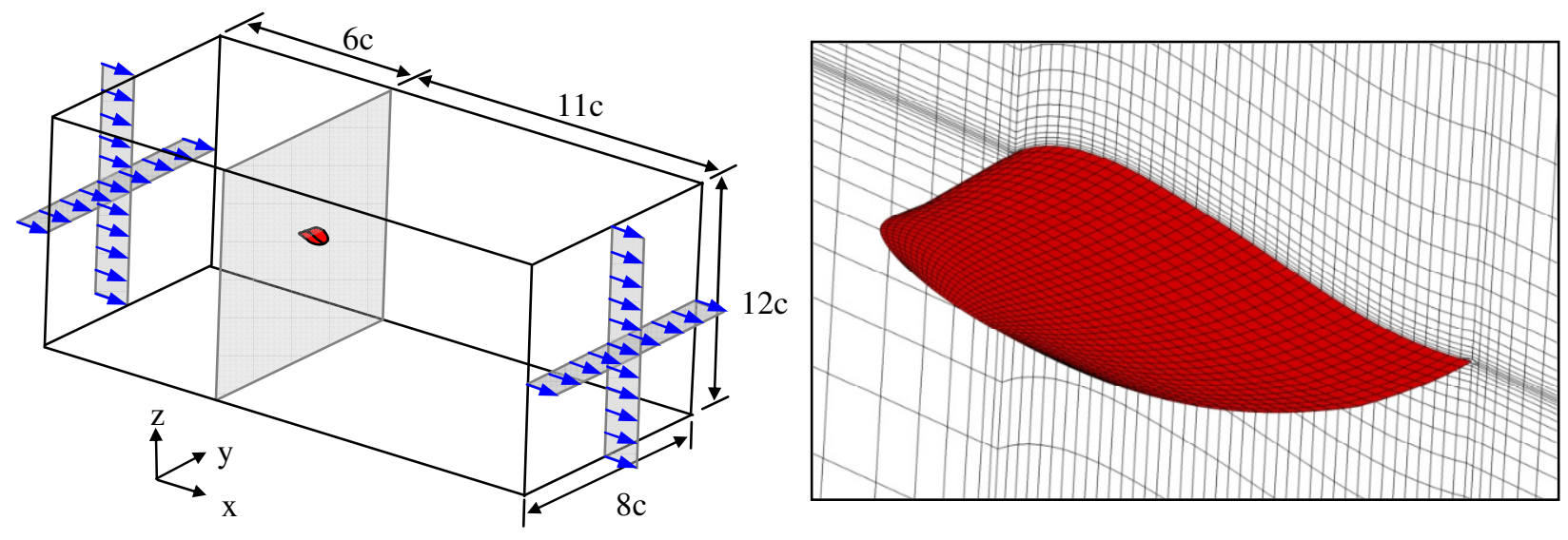

Figure 3. CFD computational domain (left) and detail of mesh near wing surface (right). 


\section{B. Finite Element Analysis}

As discussed above, the carbon fiber regions of the MAV wing are built excessively thick out of consideration for modeling simplifications. As such, only the membrane regions of the wing are modeled with the unstructured triangular mesh seen in Figure 4. The nodes that lie on the perimeter of this mesh are given a zero-displacement boundary condition. Several nonlinearities are included in the finite element formulation: large displacements (both in-plane and out-of-plane motions), finite strains, and redistribution of non-conservative pressure loads due to membrane deformation ${ }^{16}$. Material nonlinearities are ignored for the current work. While latex rubber is technically a hyperelastic solid (the dependence of the strain energy upon the invariants of the deformation tensor ${ }^{17}$ causes a nonlinear stress-strain curve), the strain levels that accumulate are assumed to be small enough (this is validated below) to warrant a linear stress-strain relationship: Hooke's law is used here.

The linear stress stiffening effect of the membrane's pre-tension must also be accounted for. As discussed above, VIC is used to measure the pre-strain in the membrane. This experimental data field is sent through a moving average smoother, and then plugged into the structural finite element model. Hooke's law is used to convert these pre-strains into pre-stresses.

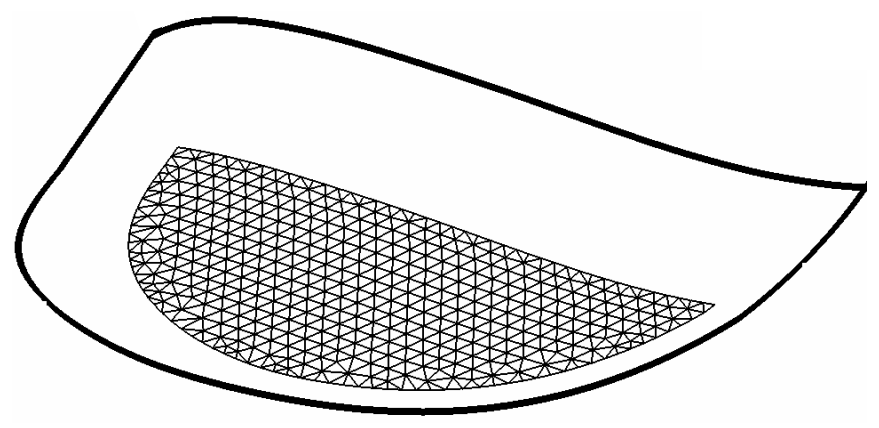

Figure 4. Finite element mesh of membrane wing skin.

\section{Aeroelastic Coupling}

The steady fluid structure interaction of the flexible MAV wing is computed in the following manner:

1) Solve the 3-D, incompressible viscous Navier-Stokes equations for the steady laminar flow field, using the CFD grid shown in Figure 3.

2) Interpolate the computed wing pressures onto the FEA grid shown in Figure 4.

3) Solve for the resulting wing displacements using the structural membrane model.

4) Interpolate the displacement onto the MAV wing of the CFD grid.

5) Re-mesh the CFD grid using a master/slave moving grid scheme ${ }^{7}$.

6) Repeat steps 1-5 until convergence is achieved: less than $0.1 \%$ change in maximum displacement.

Less than ten iterations are usually adequate for simulations at moderate angles of attack $\left(3^{\circ}<\alpha<18^{\circ}\right)$, though up to twenty may be needed for angles outside of this range. Step 1 requires between 150 and 250 sub-iterations, while step 3 can typically converge within 10 sub-iterations.

\section{Results and Discussion}

\section{A. Membrane Pre-Strain}

The amount of pre-strain injected into the membrane skin represents an aeroelastic tailoring problem. If the liftto-drag ratio (L/D) is an important design metric, then a taught membrane is preferred. This will prevent the drag penalty that ensues with excessive wing deformation. If large longitudinal static stability is required, then the membrane should be slack. For the current work, a moderate amount of pre-strain is used, by stretching a square of latex rubber ( $150 \mathrm{~mm}$ sides) biaxially: $4 \mathrm{~mm}$ in both the chordwise and the spanwise directions. The taught membrane is then adhered to the wing surface and VIC is used to measure the pre-strain fields, shown in Figure 5. The anisotropy of the tension is clearly evident. A state of isotropic biaxial tension (with no shear) is very difficult to obtain: the size of the taught membrane sheet is not significantly larger than the MAV wing surface, and thus the pre-strain is distorted by end effects. Transferring a state of isotropic tension in a flat membrane to the cambered wing surface provides further complications. As such, the pre-strain fields in the membrane wing are used in a passive sense: the general magnitude of the pre-strain can be controlled, but not (with current MAV fabrication methods) the distribution. 


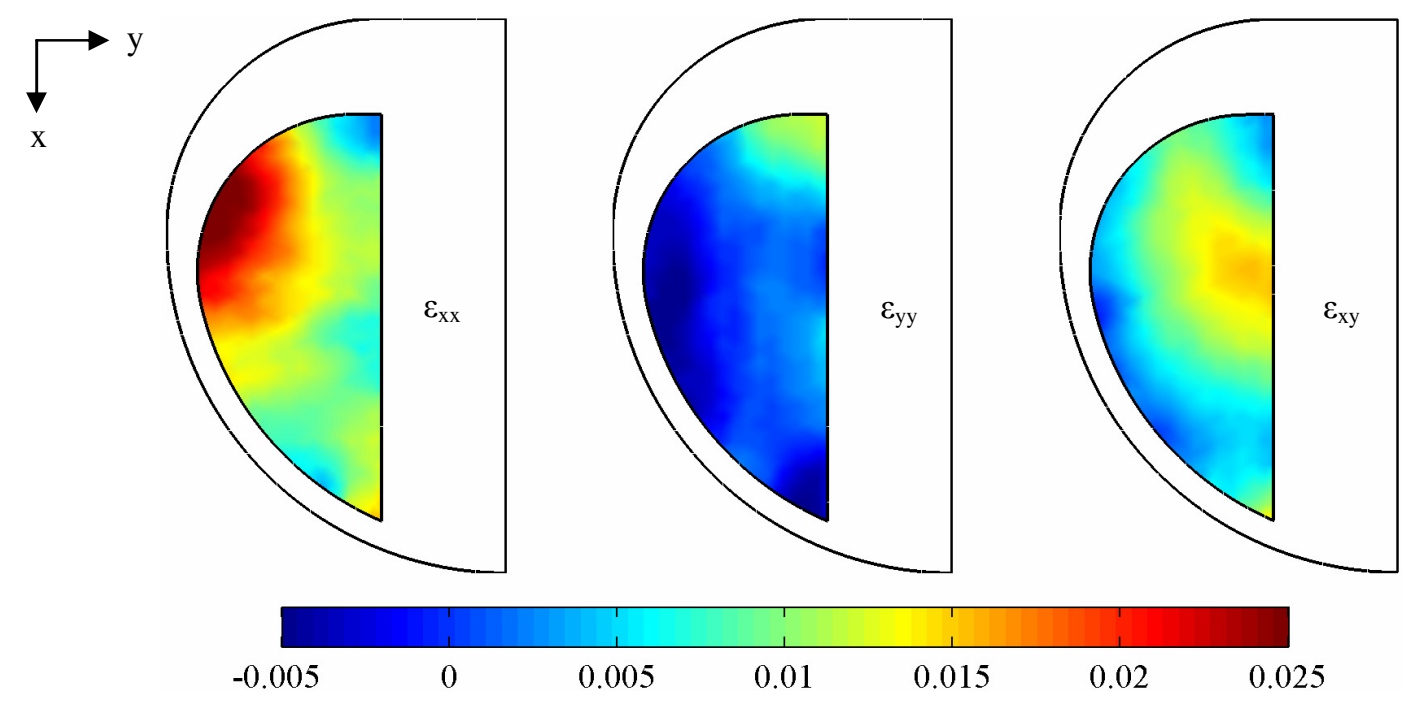

Figure 5. Chordwise (left), spanwise (center), and shear (right) membrane pre-strain fields.

\section{B. Structural Model System Identification}

Calibration of the structural model is done by applying a known load to the taught membrane MAV skin, and tuning the material parameters to achieve suitable correspondence between numerical and experimental deformation fields (Figure 6). Weights are hung from the end of a thin string glued to the center of the membrane skin. Numerically, this is modeled as a transverse point load. Two material properties are needed: the elastic modulus and the Poisson's ratio. The latter is fixed at 0.5 , the commonly accepted value for incompressible rubbers ${ }^{17}$. Two separate values for the modulus were found to provide the best fit to the data. $3 \mathrm{MPa}$ was used to convert the prestrains of Figure 5 into stresses, while $7 \mathrm{MPa}$ was used for the calculation of stresses that developed due to the transverse point load. Theoretically, these two values should be equal: errors in the calculated pre-strain (a particular problem with such small strains in the presence of substantial rigid body motions: adhering the latex to the wing) or hyperelastic effects may cause the difference.

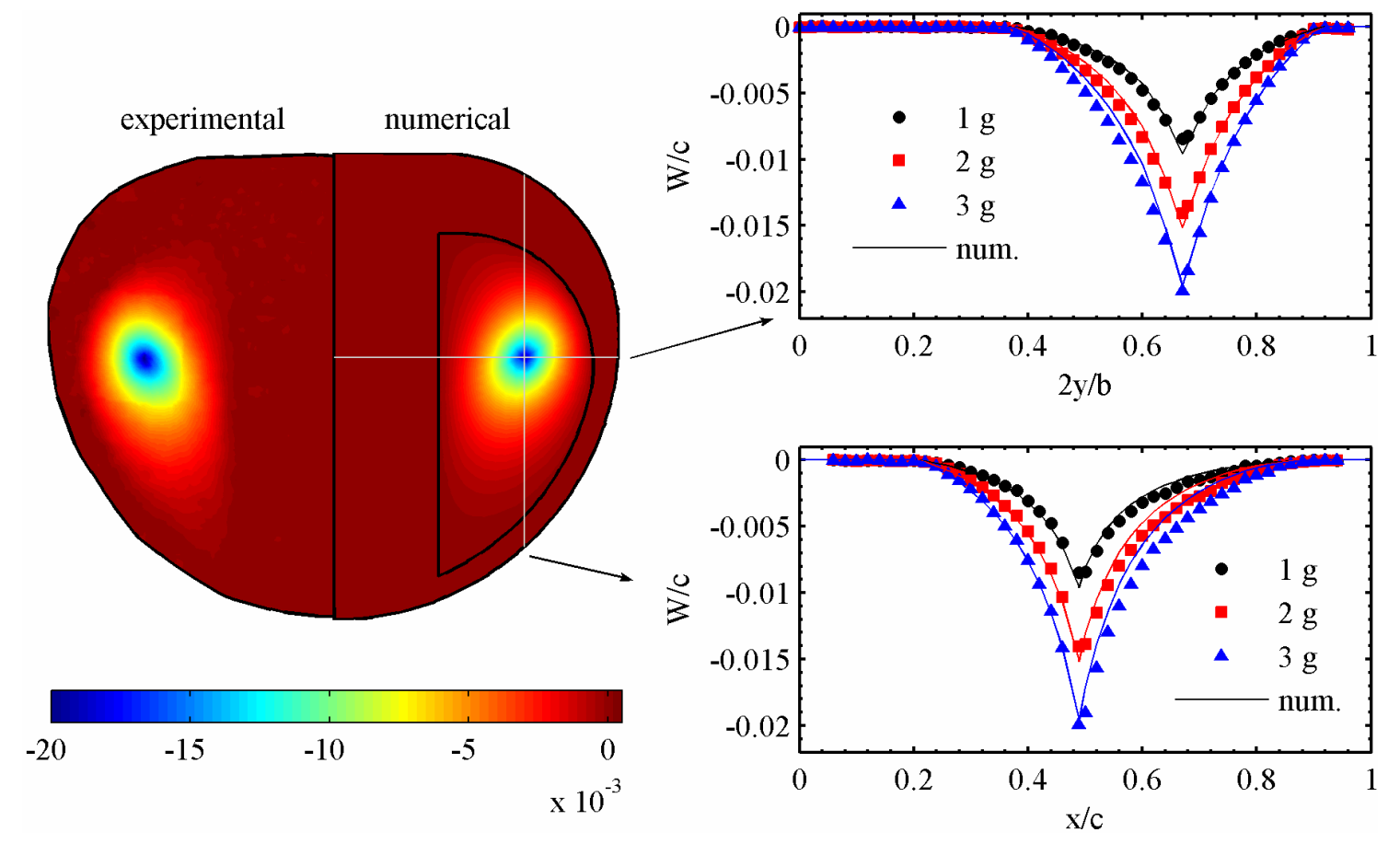

Figure 6. Normalized transverse displacements due to 1, 2, and 3 gram weights hung from a string glued to the center of the membrane wing. 


\section{Validation of Wing Deformation}

With the structural model properly tuned, we turn now to the static aeroelasticity of the membrane wing. Six elastic quantities can be measured/computed: three displacements (chordwise $\mathrm{U}$, spanwise $\mathrm{V}$, and transverse $\mathrm{W}$ ) and three plane strains. The transverse displacements and chordwise strains are thought to have the greatest effect upon the aeroelasticity, and will be the focus of the following discussion. The strain fields given below are as a result of the aerodynamic loading, and do not include the pre-strain of Figure 5.

The measured displacement field at $0^{\circ}$ angle of attack (Figure 7) is entirely confined to the membrane skin, validating the assumption of a rigid carbon fiber perimeter. The transverse displacements are relatively small (almost three orders of magnitude less than the root chord), though still affect the aerodynamics of the wing. At this low angle of attack, the magnitude of the deformation field is well predicted by the model, though the location of the maximum displacement (corresponding to the camber of the wing) is computed far forward of the measured location. This is likely due to flow separation on the underside of the wing, at the leading edge. Experimental flow visualization data ${ }^{18}$ indicates that the underside of the wing is mostly separated (burst bubble), whereas the computational data shows a separation bubble ranging from the leading edge to the quarter-chord. This would indicate that the model erroneously predicts a favorable pressure gradient aft of the quarter-chord, whereas the actual location of the peak pressure is probably farther aft. This would also explain the incorrectly predicted region of negative deformation towards the trailing edge: the computed pressures are too low in this location.

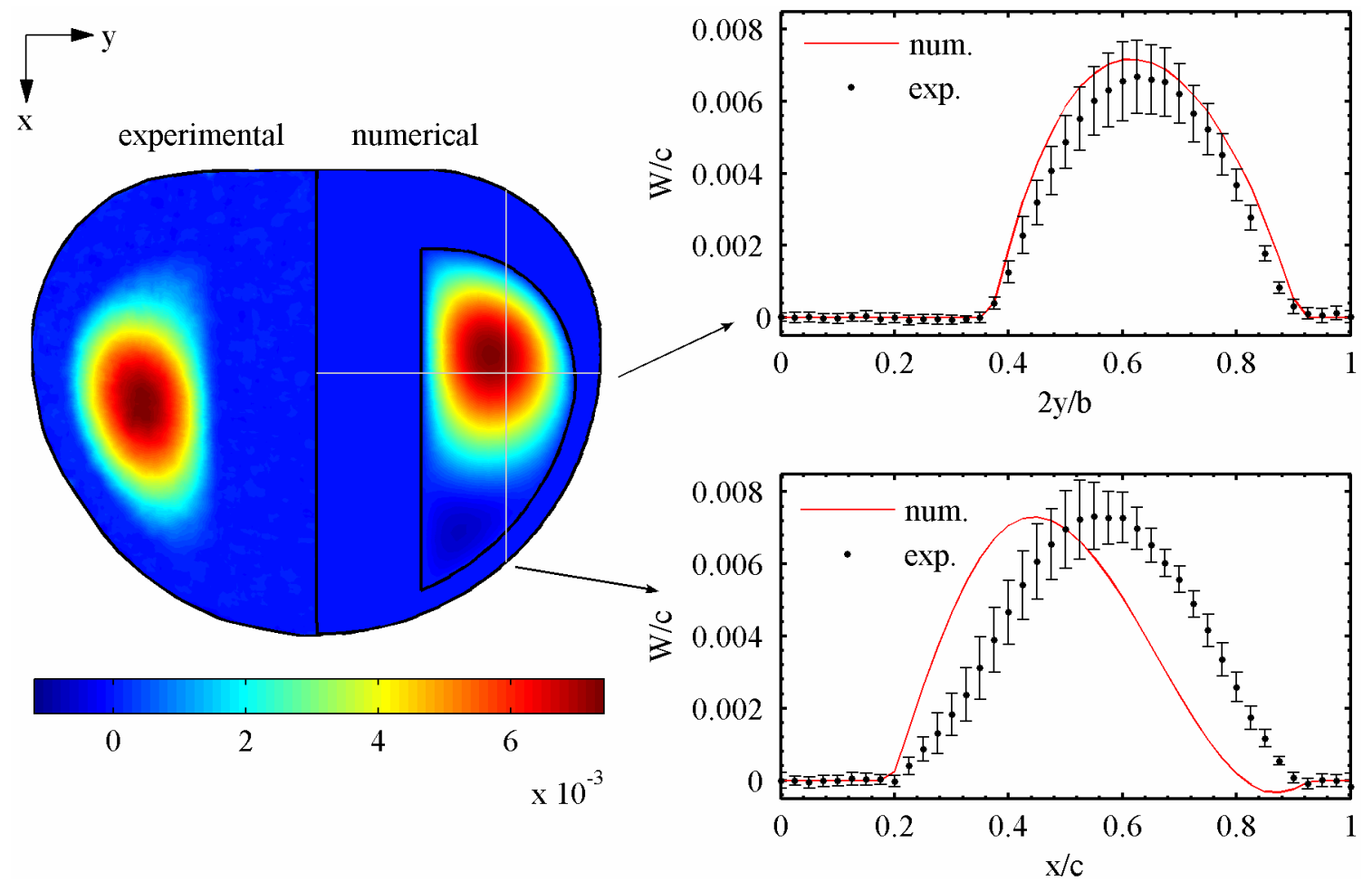

Figure 7. Normalized transverse displacements, $\alpha=0^{\circ}, U_{\infty}=13 \mathrm{~m} / \mathrm{s}$.

The error bars in the displacement measurements (Figure 7) are estimated statistically (from ten measurements, spaced 1 second apart). At $0^{\circ}$ angle of attack, the peak uncertainty range is $0.2 \mathrm{~mm}$, though the resolution error of the VIC system is estimated at $0.05 \mathrm{~mm}$ (found from the camera's field of view and pixel recording resolution ${ }^{14}$ ). The large displacement error bars are a result of unsteady flow: at low angles, the membrane wing experiences a self-excited vibration (visible in the wind tunnel). The VIC system used in this work is incapable of characterizing this vibration (estimated by Lian et al. ${ }^{7}$ to be moving at $100 \mathrm{~Hz}$ ): image sampling frequencies cannot exceed $2 \mathrm{~Hz}$. Both the flow and structural solver assume steady flow conditions, perhaps indicative of the mixed predictive capability of the aeroelastic model at this angle. The addition of a turbulence module to the aeroelastic simulation may also increase the fidelity of the model at this angle of attack.

The measured chordwise strain field at $0^{\circ}$ (Figure 8 ) is similarly small, slightly above the resolution limit of the VIC system $(\sim 1000 \mu \varepsilon)$. As such, the measured membrane strain field is fairly noisy. Furthermore, any flexural strains that may develop in the excessively stiff carbon fiber wing skeleton are sure to fall below the resolution of 
the VIC system: strain measurements in this region are only noise. The model over-predicts the magnitude of the strain in the membrane, though is able to capture the trends: a lobe of high extensional chordwise strain towards the membrane/weave boundary at the leading edge, low strain levels towards the trailing edge. Large strain gradients/magnitudes are measured at the border of the MAV wing: the VIC system has difficulties in computing the strain fields directly up to the edge of the area of interest, and are erroneous.

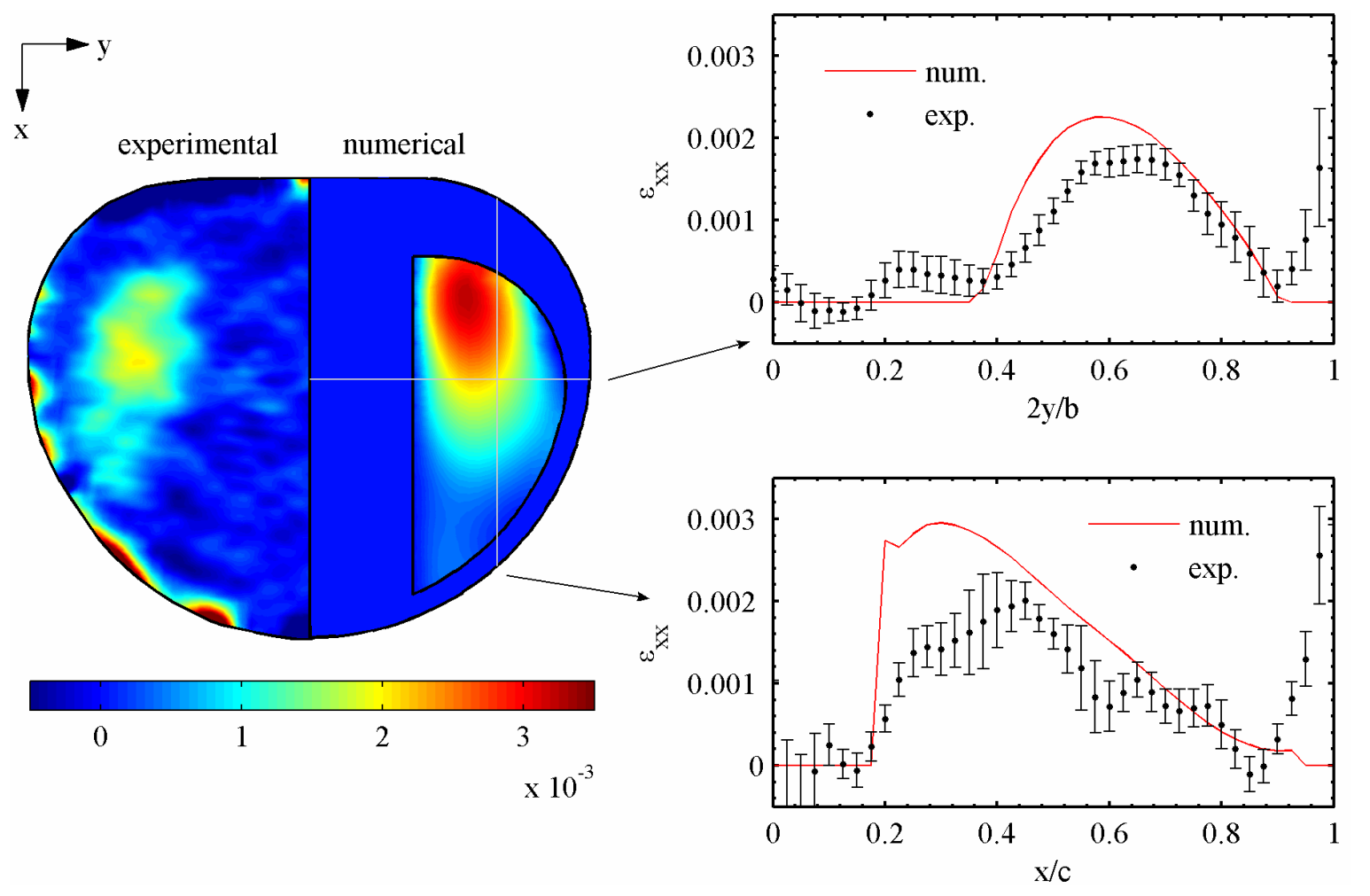

Figure 8. Chordwise strains, $\alpha=0^{\circ}, \mathrm{U}_{\infty}=13 \mathrm{~m} / \mathrm{s}$.

The displacements at $12^{\circ}$ angle of attack (Figure 9) are larger than before (two orders of magnitude less than the root chord). Both the magnitude and the location of the peak membrane displacement show good correspondence between experiment and simulation at $12^{\circ}$. The model's high predictive capability at this moderate angle of attack is likely due to the smooth flow field: the flow over the bottom surface of the MAV wing is completely attached. As such, both the experimental and numerical transverse displacements are completely positive. The magnitude of the estimated displacement error is significantly smaller than that seen at the smaller angle: the flow is largely steady at $12^{\circ}$, and the error bars now reflect the inherent uncertainty/repeatability of the VIC system. The average displacement error bar is $0.05 \mathrm{~mm}$ long, consistent with the estimated resolution error of the VIC. The chordwise strain field at $12^{\circ}$ (Figure 10) is similarly well-predicted by the aeroelastic model. The lobe of high extensional chordwise strain is measured slightly aft of the membrane/weave boundary, while the model predicts peak strain directly on the boundary. The significantly higher strain levels $\left(0.7 \%\right.$ at $12^{\circ}$, up from $0.2 \%$ at $\left.0^{\circ}\right)$ provide a cleaner strain reading, though the VIC system again produces erroneous results at the edge of the MAV wing.

At $30^{\circ}$ angle of attack, the wing is in the post-stall regime: the flow is completely separated over the top surface of the wing. The separated flow is very unsteady, as indicated by the large displacement error bars in Figure 11. Nevertheless, the time-averaged wing displacement field is well-predicted by the steady aeroelastic model, in both the location and the magnitude of the peak displacement $(\sim 2 \mathrm{~mm})$. The predicted strain field is less accurate at this angle. The measured chordwise strain (Figure 12) is completely extensional, with the familiar lobe of high strain towards the leading edge. The model accurately predicts the lobe, but indicates a region of negative strain towards the trailing edge. This is probably Poisson strain, ostensibly due to high extensional strain in the span direction. Wrinkling phenomena (latex membrane cannot sustain a compressive stress ${ }^{17}$ ) may also be present, as the measured extensional strain in this region is very close to 0 . As a wrinkling module is not built into the finite element analysis, the model will instead predict membrane compression. A wrinkling pattern is not clearly visible from the measured displacement field (Figure 11) however, though is reported by Fink ${ }^{8}$ for stalled membrane wings. 


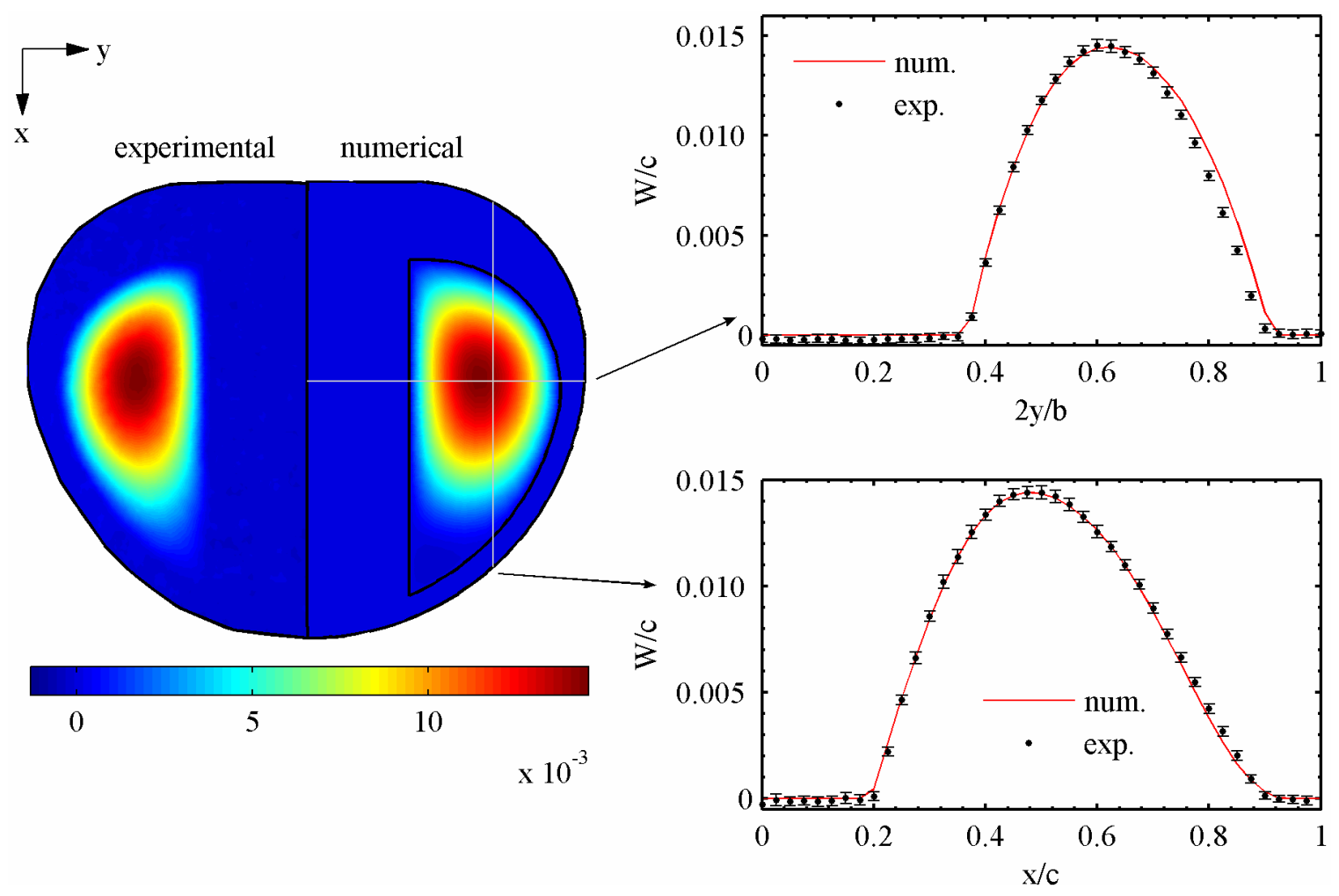

Figure 9. Normalized transverse displacements, $\alpha=12^{\circ}, U_{\infty}=13 \mathrm{~m} / \mathrm{s}$.

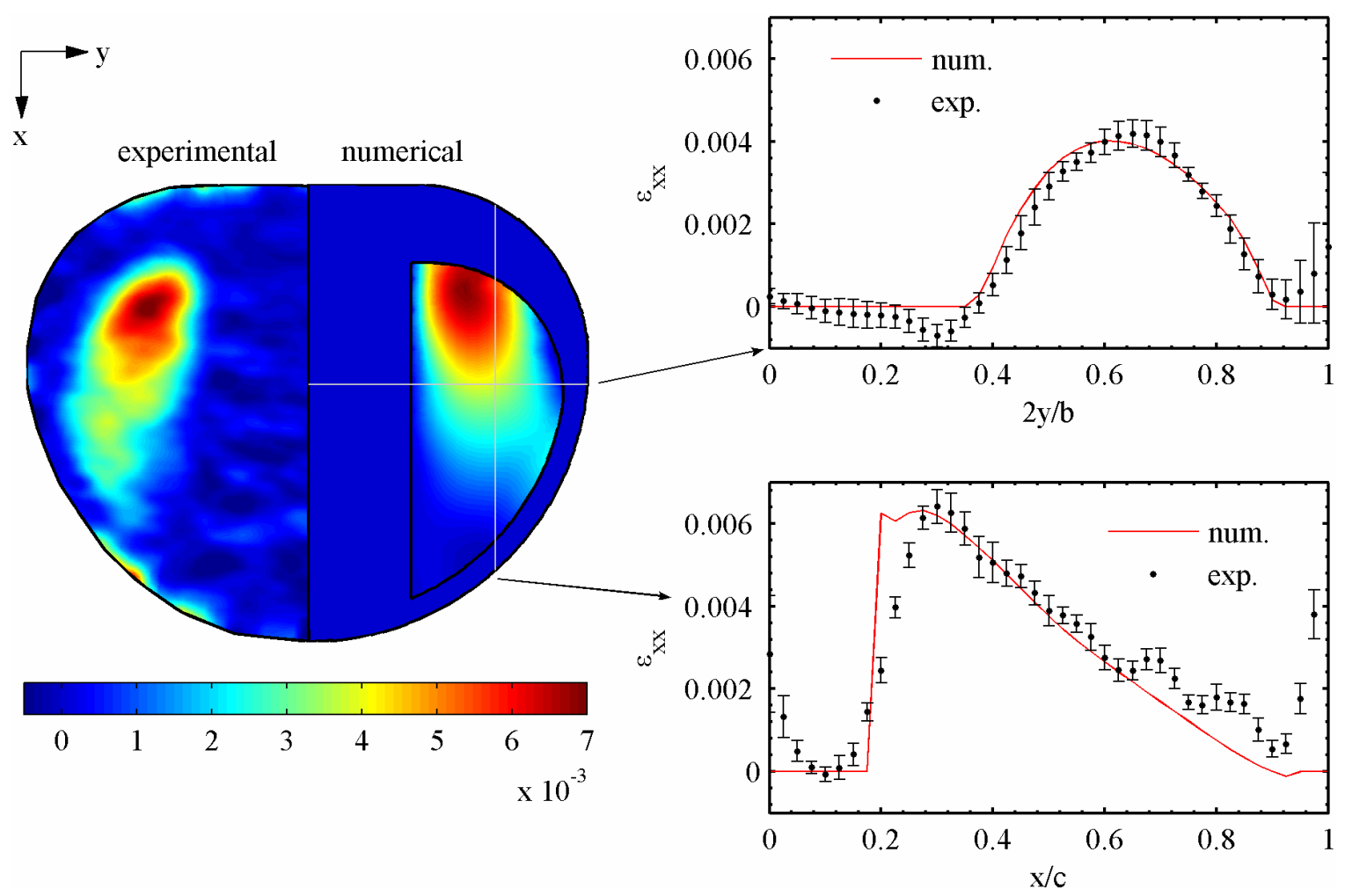

Figure 10. Chordwise strains, $\alpha=12^{\circ}, U_{\infty}=13 \mathrm{~m} / \mathrm{s}$. 


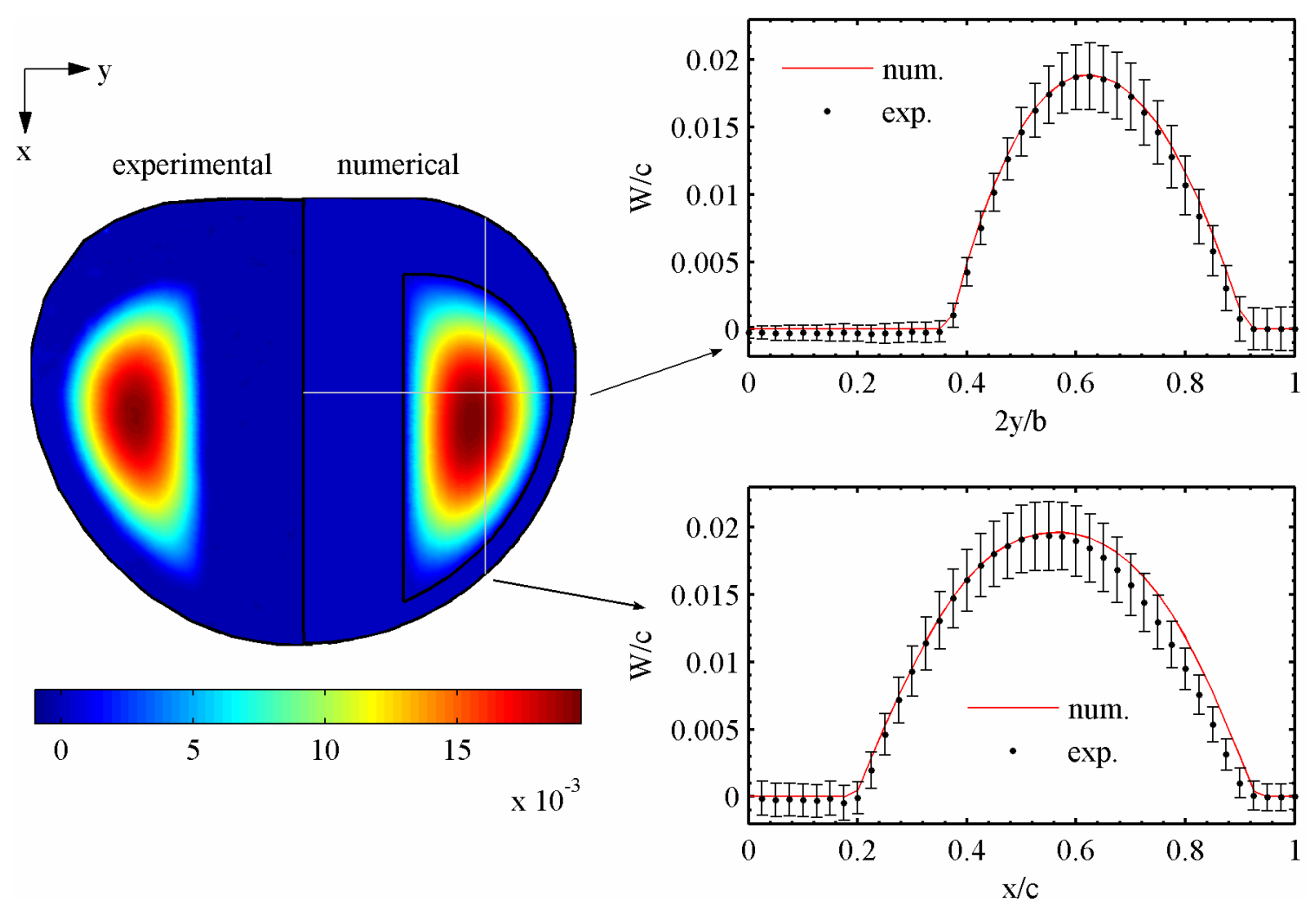

Figure 11. Normalized transverse displacements, $\alpha=30^{\circ}, U_{\infty}=13 \mathrm{~m} / \mathrm{s}$.

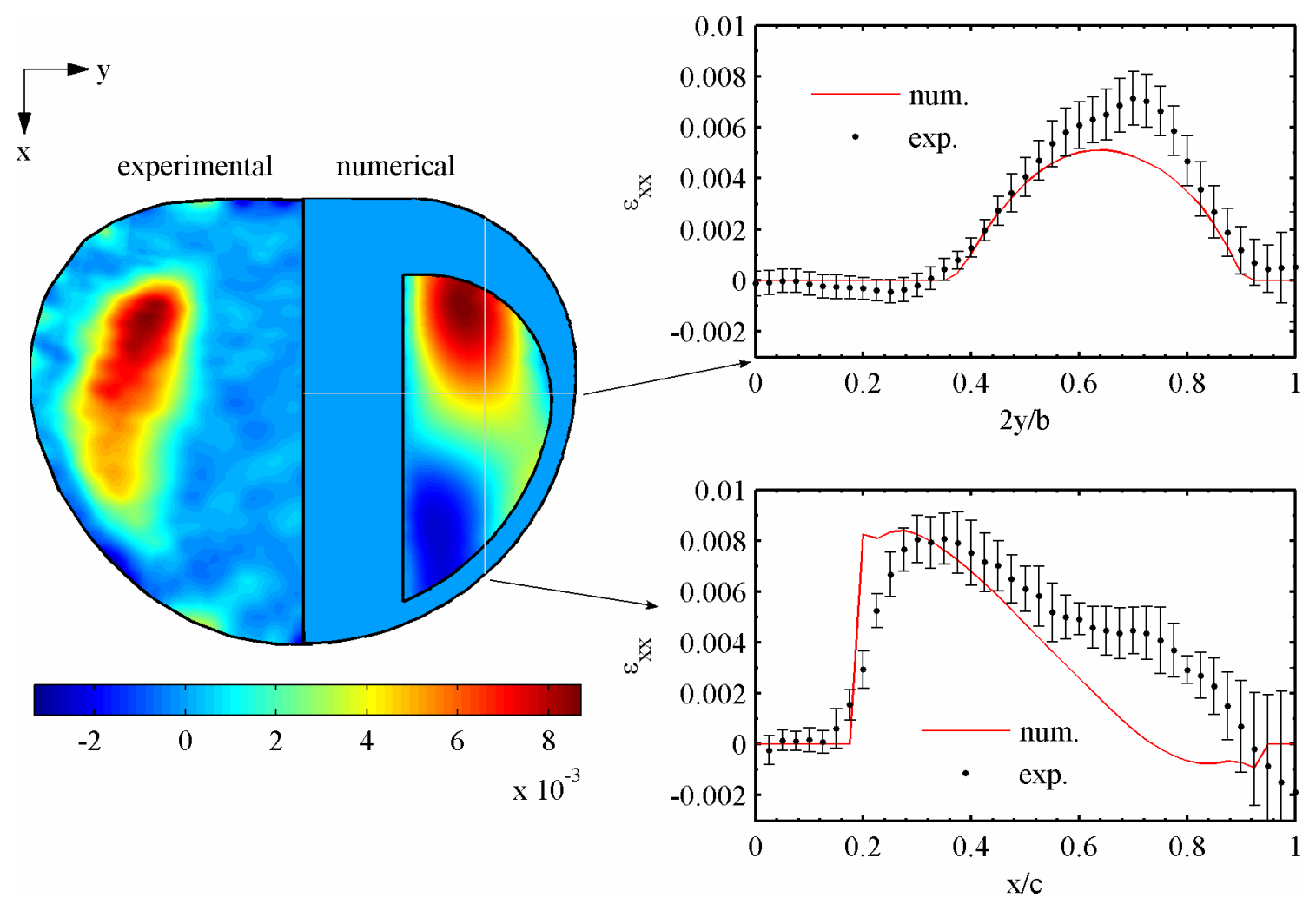

Figure 12. Chordwise strains, $\alpha=30^{\circ}, U_{\infty}=13 \mathrm{~m} / \mathrm{s}$. 
The maximum normalized displacement and the normalized location of this peak as a function of angle of attack can be seen in Figure 13. The nonlinear behavior of the membrane deformation is clearly evident: up to the stall angle, the lift is linear with incidence (some low aspect ratio wings display a nonlinear lift curve due to interference effects between the tip vortex swirling system and the wing circulation, but this is not the case with the UF MAV wing shown in Figure 1), while the transverse membrane inflation is not. This is a direct effect of the moderate amount of pre-strain (Figure 5) in the membrane wing. Larger pre-strains will lead to a more linear inflation curve, though the peak displacement will be lower than that seen in Figure 13. A completely linear stress-stiffening model could then be used, as is the approach of Sugimoto ${ }^{6}$. The size of the error bars indicates a region of steady flow at moderate angles of attack; between $6^{\circ}$ and $21^{\circ}$ (the latter is the approximate stall angle). For all angles, the aeroelastic model is capable of predicting the magnitude of the peak wing displacements to within experimental error bars. The initial de-cambering of the wing with the onset of stall is not predicted by the model, however.
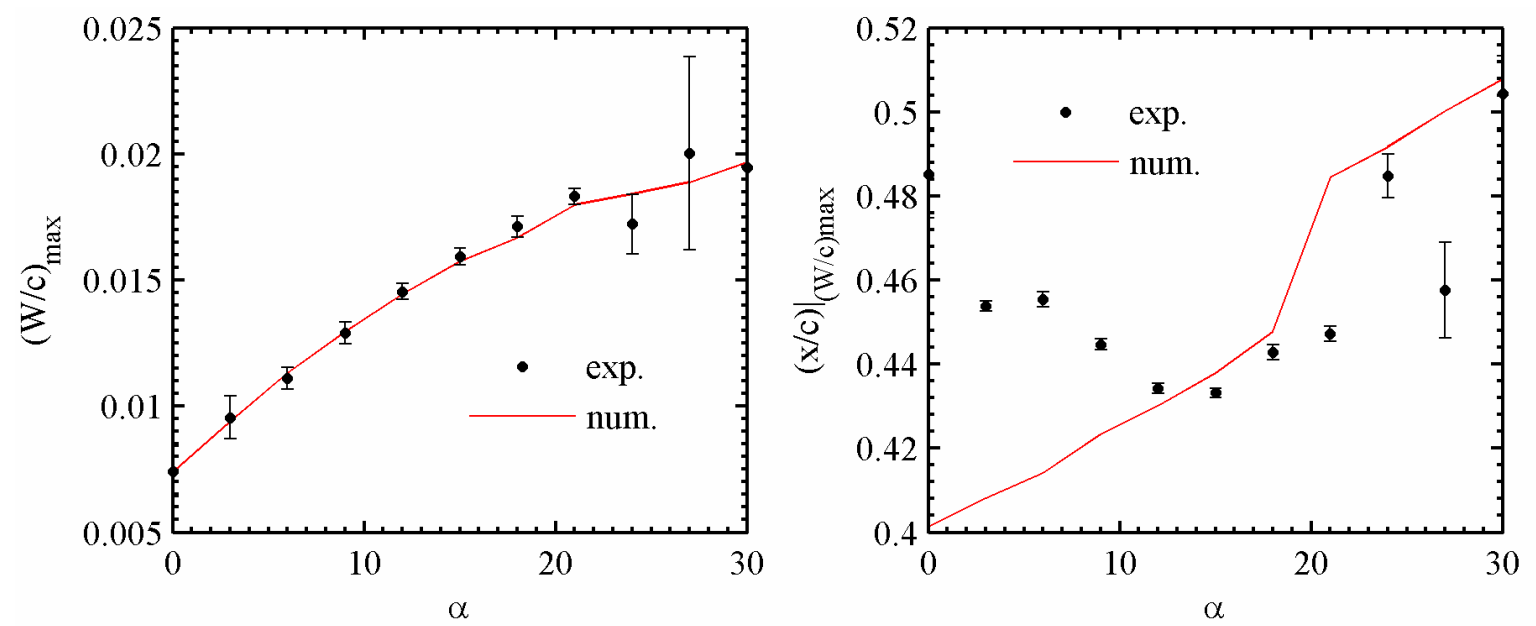

Figure 13. Normalized peak displacement (left) and the normalized location of the peak (right), $U_{\infty}=13 \mathrm{~m} / \mathrm{s}$.

The model is less successful at predicting the location of this peak displacement. The model places the camber $18 \%$ closer to the leading edge than the measured location at $0^{\circ}$. As the incidence increases, the model predicts that the camber moves aft, while the measured trend is opposite: towards the leading edge. As discussed above, this is a result of the model's inability to characterize the separated flow patterns on the lower wing surface. The experimental and numerical data trends cross over at $12^{\circ}$ angle of attack, wherein the experimental trend reverses direction. This reversal is thought to coincide with the flow completely attaching itself to the lower wing surface. Above $12^{\circ}$, the aeroelastic model is able to predict the camber location with suitable accuracy. The elastic energy (the energy density is computed at each node from the strains, and then integrated over the wing) within the wing can be seen in Figure 14. As before, only the strains that accumulate during flight are considered, not the prestrains. Though the model's predictive capability is suitable at low angles of attack, the strain energy is slightly over-predicted. This is possibly due to the low strains that develop within the membrane, which are (as noted in Figure 8) very close to the strain resolution of the VIC system. The model is able to predict the strain energy up to a moderate angle of attack $\left(\sim 9^{\circ}\right)$; after which it significantly under-predicts the energy. This is most likely due to noise in the strain readings. Integrating over the entire wing provides an appreciable energy contribution from the strain spikes at the wing boundary and the noise in the carbon fiber regions. The erroneous prediction of compressive strain towards the trailing edge of the membrane wing (Figure 12) may also lead to the poor correspondence at high angles of attack.

\section{Validation of Longitudinal Aerodynamic Coefficients}

The coefficients of lift, drag, and pitching moment are given in Figure 15 and Figure 16, for both the membrane wing considered above, as well as a rigid wing. The latter has the same camber, dihedral, and planform shape as the un-deformed membrane wing. The rigid wind tunnel model is built from 5 layers of bi-directional carbon fiber, and is constructed upon the same $\mathrm{CNC}$ mold as used for the membrane wing.

The computational fluid dynamics scheme detailed above is able to accurately predict the pre-stall lift and drag over a rigid MAV wing: computed trends consistently fall within the experimental error bars. The lift over the rigid wing is over-predicted by $11.3 \%$ at $3^{\circ}$, and under-predicted by $1.24 \%$ at $18^{\circ}$. The drag is over-predicted by $8.51 \%$ at 
$3^{\circ}$, and over-predicted by $11.9 \%$ at $18^{\circ}$. The stalling angle is under-predicted by $2^{\circ}$. As the wing displacements in the previous section are relatively small, so are the changes in aerodynamic performance due to the membrane inflation. As expected, the lift, drag, and lift slope all increase (pre-stall). The model generally predicts a smaller increase in aerodynamic force than that measured in the wind tunnel. For example, at $18^{\circ}$ angle of attack, the model predicts a $2.67 \%$ increase in lift due to the adaptive inflation, while the experimental data indicates a $7.52 \%$ increase. Similarly, the model predicts a $2.51 \%$ drag penalty, but a $7.58 \%$ penalty is measured at $18^{\circ}$. The aeroelastic model also incorrectly predicts lower lift and drag (as compared to the rigid wing aerodynamics) in the post-stall regime, possibly due to simulated de-cambering of the membrane wing. Despite these errors in the aeroelastic simulations, the numerical data consistently lies within the experimental error bars for the membrane wing aerodynamics.

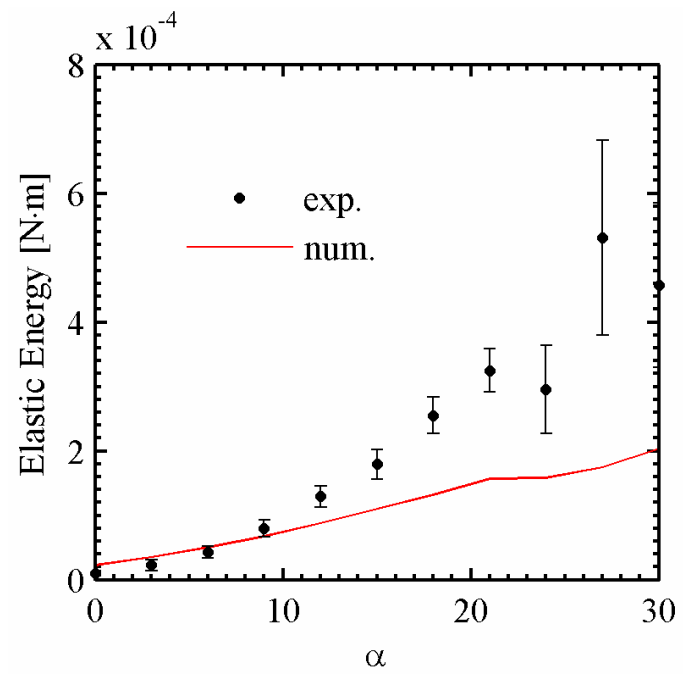

Figure 14. Accumulated strain energy within the MAV wing, $U_{\infty}=13 \mathrm{~m} / \mathrm{s}$.
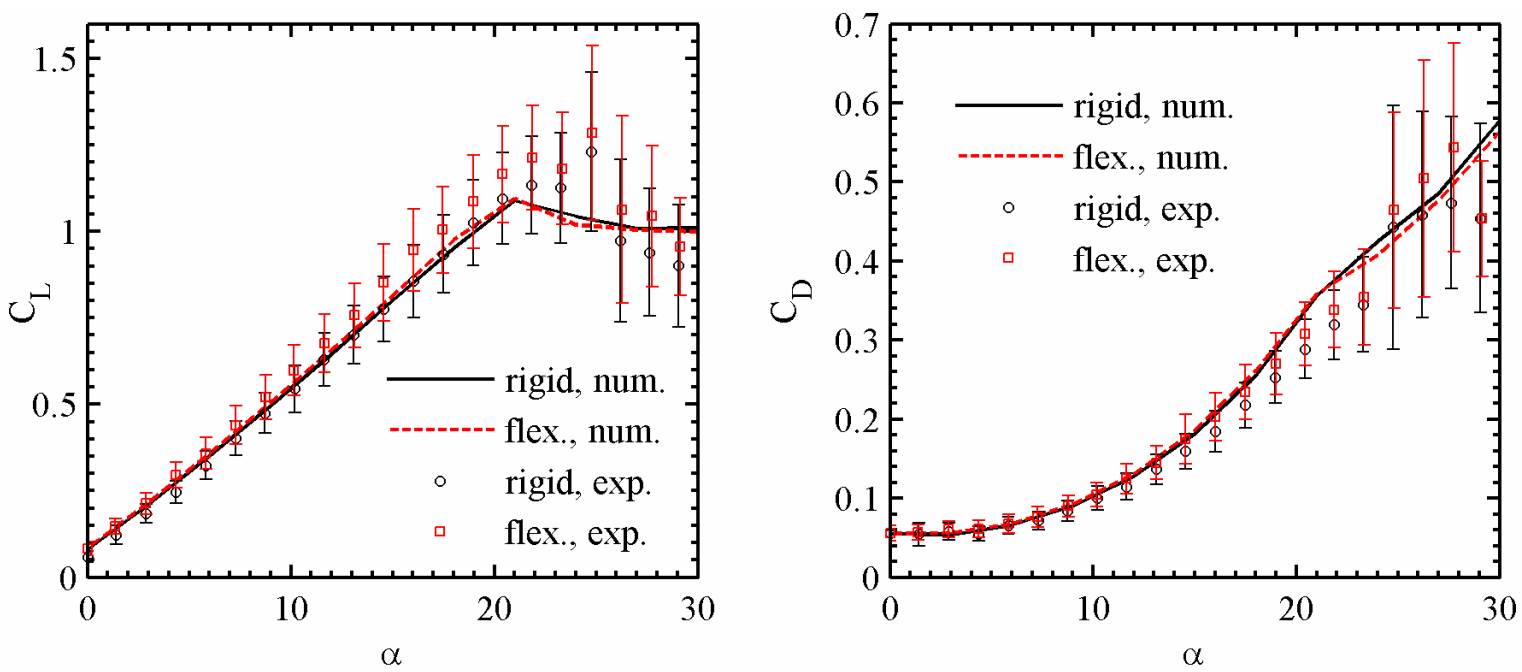

Figure 15. Lift and drag for both a rigid and membrane MAV wing, $U_{\infty}=13 \mathrm{~m} / \mathrm{s}$.

The longitudinal static stability is given in Figure 16, where the pitching moment is measured about the leading edge. The nose-down moment of the rigid MAV wing is consistently over-predicted by the CFD model $(49.6 \%$ at $18^{\circ}$ angle of attack); though it still falls within the relatively large error bars of the experimental data. The ability of the rigid CFD model to accurately predict lift but not pitching moment is analogous to the data of Figure 13: the magnitude of the deformation is well-predicted, but not necessarily the distribution of the displacement. The adaptive membrane inflation increases the nose-down pitching moment by increasing the force on the wing. At moderate angles, the moment arm is increased as well (Figure 13). This increases the negative slope of the pitching 
moment curve and the static margin of the wing, representing the major benevolent characteristics of the adaptive wing. Stability concerns are a primary target of design improvement from one generation of micro air vehicles to the next. The range of flyable CG locations on a MAV is generally a few millimeters long: meeting this requirement is a strenuous weight management challenge. As before, the aeroelastic model is able to predict the correct trend (5.36\% increase in nose-down pitching moment with the membrane wing at $\left.18^{\circ}\right)$, but under-predicts the magnitude of the change $\left(23.7 \%\right.$ measured increase at $\left.18^{\circ}\right)$.

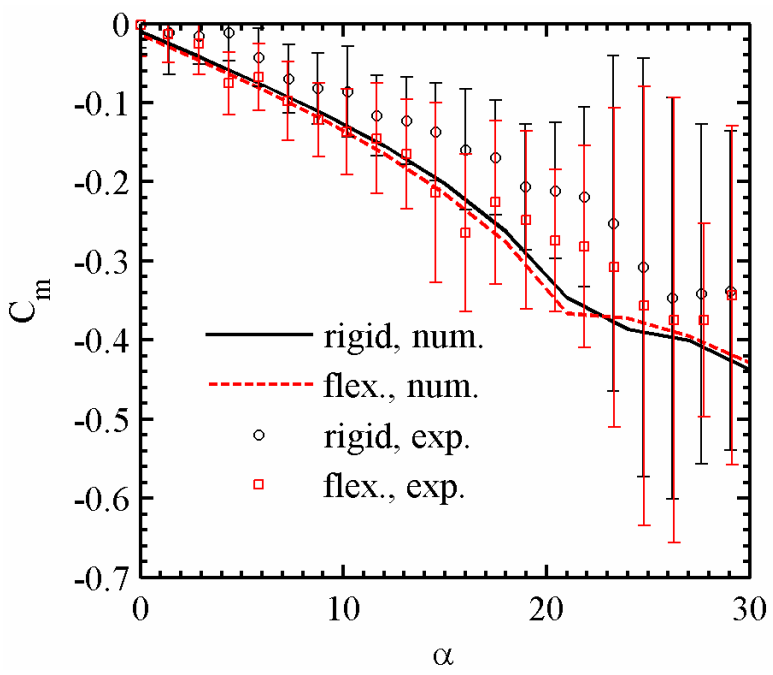

Figure 16. Longitudinal static stability for both a rigid and membrane MAV wing, $U_{\infty}=13 \mathrm{~m} / \mathrm{s}$.

\section{E. Computed Pressure Distributions}

As the previous sections have provided confidence in the aeroelastic model's ability to predict deformed wing shapes, accumulated membrane strains, and aerodynamic forces/moments, attention is now turned to numerical data that cannot (with the current experimental apparatus) be verified in the laboratory. The simulated pressure distributions over the top and bottom wing surfaces for both the rigid and the membrane wing are given in the following figures, for $0^{\circ}$ and $15^{\circ}$ angle of attack. For both angles, the low pressure region on the top surface towards the wing tip is evident, caused by the core of the tip vortex swirling system. The strength of this system is due to the low aspect ratio nature of the wing, and is seen to increase with angle of attack. The pressure drops at the tips of the membrane wings are slightly smaller than those found in the corresponding rigid wings (from -295.6 Pa to -287.9 Pa at $15^{\circ}$ ). This would perhaps indicate that the strain energy in the adaptively inflated membrane is removing energy from the vortex structure in the wake, thus decreasing the induced drag. This would merely be a drag redistribution, as the total drag seen in Figure 15 is clearly higher for the membrane wing. The data does indicate that the rolling instabilities associated with low aspect ratio wings may be improved with flexible membranes.

At the low angle attack $\left(0^{\circ}\right.$, shown in Figure 17 and Figure 18), flow over the rigid wing is prone to separation at a number of locations. The onset of pressure recovery over the top surface occurs at $\mathrm{x} / \mathrm{c}=0.15$, and is followed by an adverse gradient. This gradient is exacerbated by the presence of a high pressure region over the re-curved portion of the airfoil. The resulting separation bubble in this region is not typically evident in similar computations involving singly-curved wings at low angles of attack ${ }^{7}$. A second adverse pressure gradient is seen between the leading edge and the quarter-chord of the lower surface of the rigid MAV wing, leading to a large separation bubble. Figure 7 (and the associated discussion) indicates that the region of peak pressure should be located farther downstream, however. Aft of the computed bubble the flow accelerates under the re-curved are of the wing, resulting in a pressure drop.

When the inflated membrane shape is included in the flow structure at $0^{\circ}$ simulations, the tangent discontinuity in the wing surface at the membrane/weave boundary forces the flow over the top surface to decelerate (rapidly change direction). This causes the pressure spike clearly evident in Figure 18. The flow then accelerates over the inflated membrane shape (the increased camber leads to higher pressures than that seen in rigid wing computations, increasing the nose-down pitching moment and thus the static margin) before separating, as in the rigid wing case. On the bottom surface of the membrane wing, flow still separates at the leading edge, but then accelerates into the cavity of the inflated shape, resulting in a small area of lower pressure. The region beneath the inflated membrane is massively separated, until the flow again accelerates under the re-curved portion of the wing, and into the 
freestream. As on the top surface of the membrane wing, the adaptive inflation pushes the high pressure region slightly aft, improving static stability.
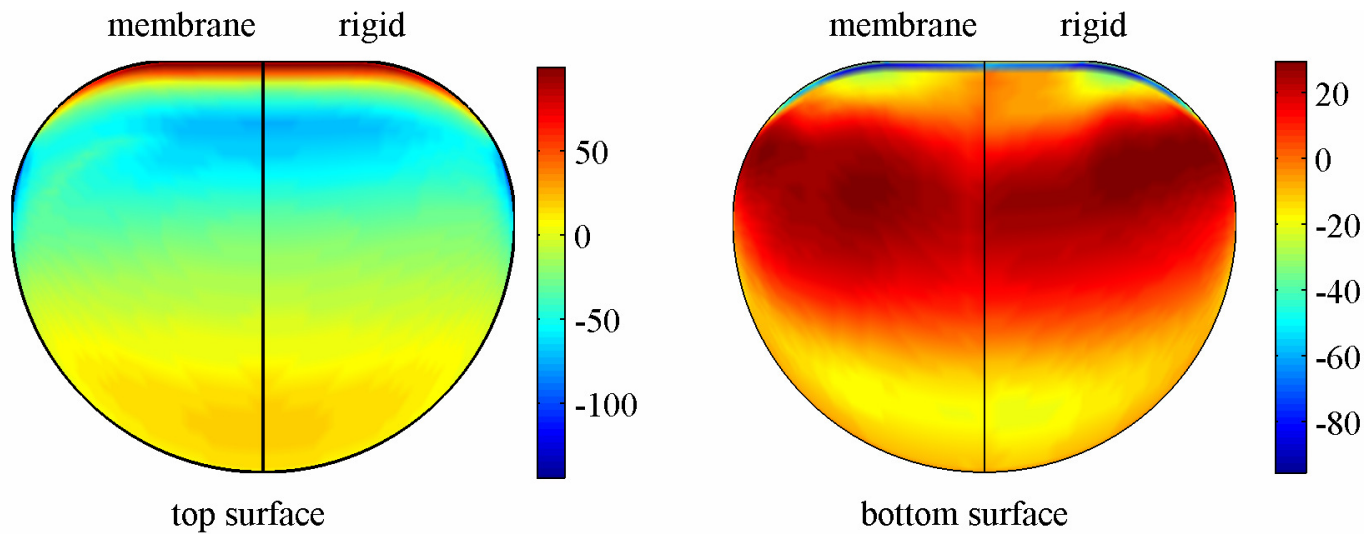

Figure 17. Computed pressure (Pa) at $\alpha=0^{\circ}, \mathrm{U}_{\infty}=13 \mathrm{~m} / \mathrm{s}$.

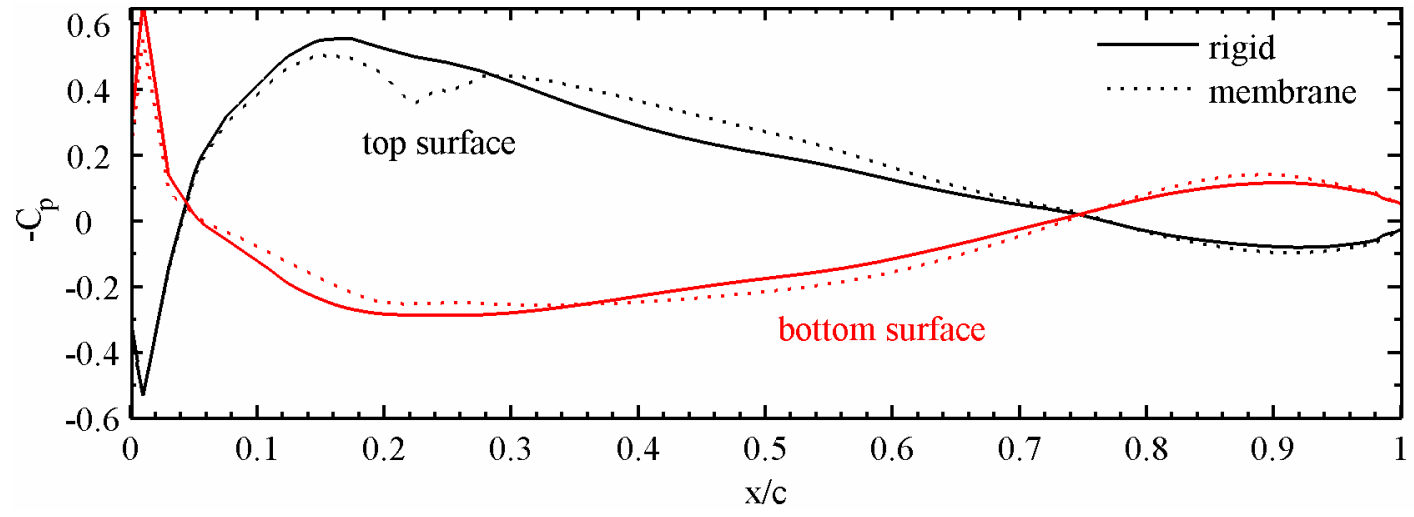

Figure 18. Computed pressure coefficients at $2 \mathrm{y} / \mathrm{b}=0.7$, at $\alpha=0^{\circ}, \mathrm{U}_{\infty}=13 \mathrm{~m} / \mathrm{s}$.

At $15^{\circ}$ angle of attack (Figure 19 and Figure 20), the favorable pressure gradient over most of the lower surface of the rigid MAV wing indicates a smoothly accelerated flow from the leading edge to the trailing edge. The flow over the top of the rigid wing remains separated, though both the size of the computed adverse pressure gradient and the increased incidence signify a larger separated zone than that seen in computations at $0^{\circ}$. For the membrane wing at $15^{\circ}$, the pressure spike on the top surface is very pronounced, as is an extended low pressure zone that develops at the membrane/weave boundary towards the trailing edge of the lower surface.

\section{Conclusion}

A series of wind tunnel tests were performed in order to validate a static aeroelastic model for low Reynolds number, low aspect ratio membrane micro air vehicle wings with adaptively inflating camber. A visual image correlation system, in conjunction with a standard strain gage sting balance, were used to measure the wing loads, displacements, and strains for a range of pre- and post-stall angles of attack. Modeling efforts included coupling a membrane finite element with geometric nonlinearities to a viscous laminar Navier-Stokes solver. A comparison of experimental and numerical deformed membrane profiles indicates poor predictive capabilities of the steady aeroelastic solver at low angles of attack: massive separation under the wing leads to unsteady flow phenomena. Good correlation in wing displacements and strains are attainable at moderate angles (where the flow is steady), and even at post-stall angles (where the flow is not). The Navier-Stokes solver is able to predict the longitudinal aerodynamics over a rigid MAV wing within experimental error for the entire $\alpha$-sweep. The aeroelastic solver correctly indicates the trends in aerodynamic performance with adaptive camber adjustment (increased lift, lift slope, and drag), though the magnitude of the shift is generally under-predicted. Turbulence effects, unsteady flow, and membrane wrinkling are all identified as candidates for upgrading the model fidelity. An examination of the computed pressure distributions identifies several key aeroelastic effects: decreased tip vortex strength, pressure spikes and flow deceleration at the tangent discontinuity of the inflated membrane boundary, and an adaptive shift of 
high pressure (on the bottom surface) and low pressure (on the top surface) regions towards the trailing edge, thereby increasing the nose-down pitching moment and the static margin.

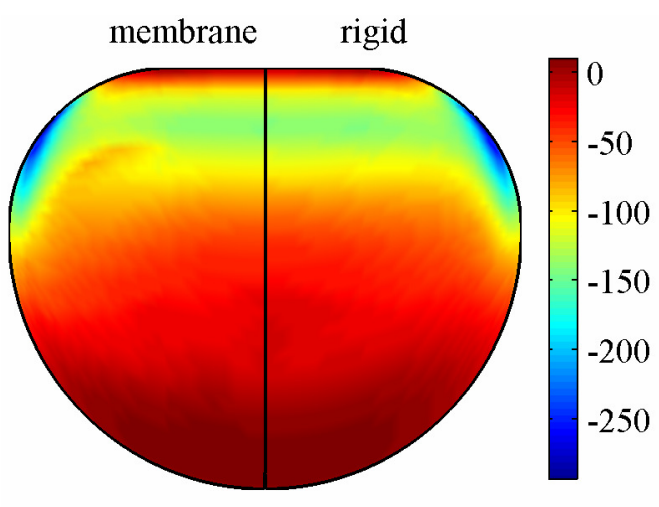

top surface

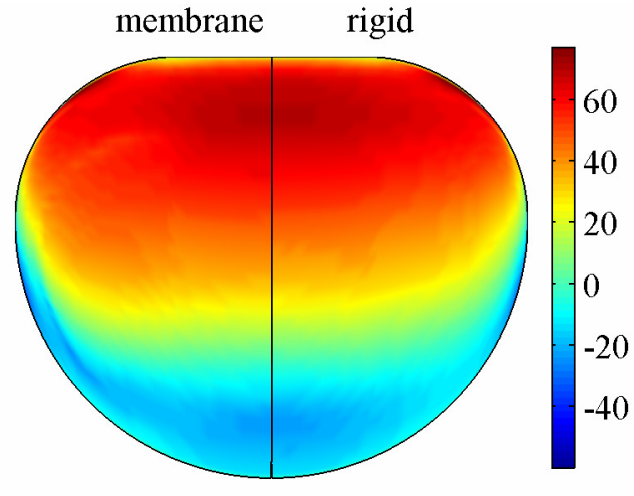

bottom surface

Figure 19. Computed pressure (Pa) at $\alpha=15^{\circ}, U_{\infty}=13 \mathrm{~m} / \mathrm{s}$.

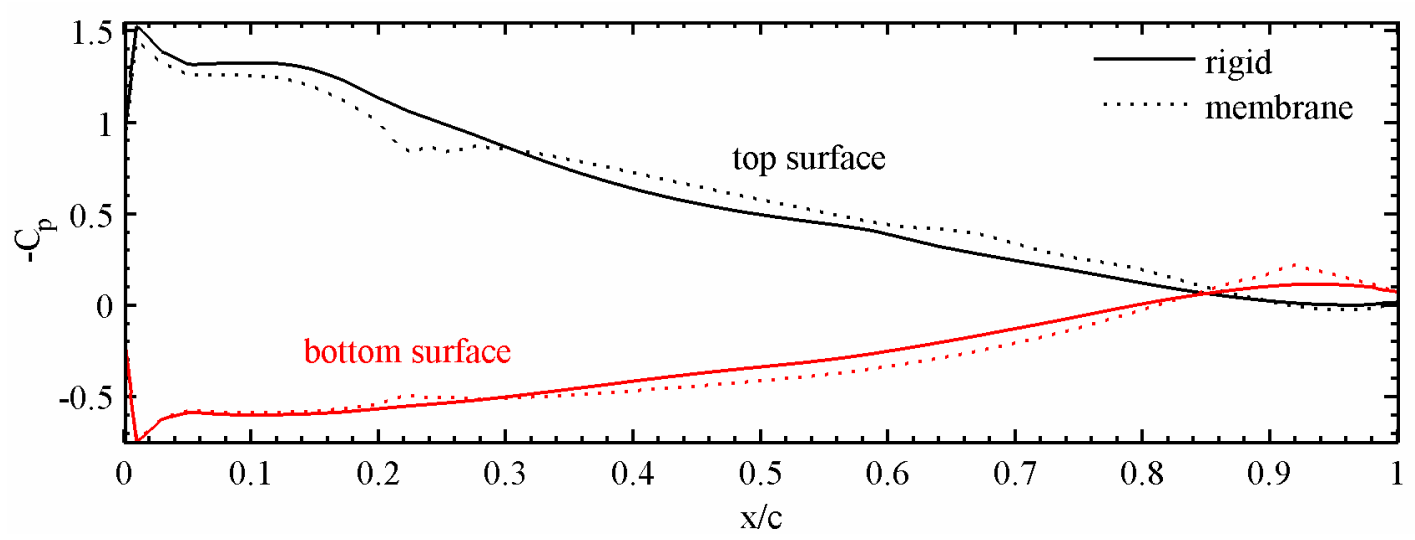

Figure 20. Computed pressure coefficients at $2 \mathrm{y} / \mathrm{b}=0.7$, at $\alpha=15^{\circ}, \mathrm{U}_{\infty}=13 \mathrm{~m} / \mathrm{s}$.

\section{Acknowledgments}

This work was jointly supported by the Air Force Research Laboratory and the Air Force Office of Scientific Research under F49620-03-1-0381 with Todd Combs, Sharon Heise, and Johnny Evers as project monitors. The authors would also like to acknowledge the technical contributions and funding of Martin Waszak at the NASA Langley Research Center.

\section{References}

${ }^{1}$ Ifju, P., Ettinger, S., Jenkins, D., Martinez, L., "Composite Materials for Micro Air Vehicles," SAMPE Annual Conference, Long Beach, CA, May 6-10, 2001

${ }^{2}$ Jackson, P., "A Simple Model for Elastic Two-Dimensional Sails," AIAA Journal, Vol. 21, pp 153-155, 1983

${ }^{3}$ Smith, R., Shyy, W., "Computation of Aerodynamic Coefficients for a Flexible Membrane Airfoil in Turbulent Flow: A Comparison with Classical Theory," Physics of Fluids, Vol. 8, pp 3346-3353, 1996

${ }^{4}$ Boudreault, R., "3-D Program Predicating the Flexible Membrane Wing's Aerodynamic Properties," Journal of Wind Engineering and Industrial Aerodynamics, Vol. 19, pp 277-283, 1985

5Jackson, P., Christie, G., "Numerical Analysis of Three-Dimensional Elastic Membrane Wings," AIAA Journal, Vol. 25, pp 676-682, 1987

${ }^{6}$ Sugimoto, T., "Analysis of Circular Elastic Membrane Wings," Transactions of the Japanese Aerodynamics and Space Sciences, Vol. 34, pp 154-166, 1991

${ }^{7}$ Lian, Y., Shyy, W., Viieru, D., Zhang, B., "Membrane Wing Aerodynamics for Micro Air Vehicles," Progress in Aerospace Sciences, Vol. 39, pp 425-465, 2003 
${ }^{8}$ Fink, M., "Full Scale Investigation of the Aerodynamic Characteristics of a Model Employing a Sailwing Concept," NASA Technical Report, TN D-4062, 1967

${ }^{9}$ Greenhalgh, S., Curtiss, H., "Aerodynamic Characteristics of a Flexible Membrane Wing,” AIAA Journal, Vol. 24, pp 541551,1986

${ }^{10}$ Galvao, R., Israeli, E., Song, A., Tian, X., Bishop, K., Swartz, S., Breuer, K., "The Aerodynamics of Compliant Membrane Wings Modeled on Mammalian Flight Mechanics," $36^{\text {th }}$ AIAA Fluid Dynamics Conference and Exhibit, San Francisco, CA, June $5-8,2006$

${ }^{11}$ Fleming, G., Bartram, S., Waszak, M., Jenkins, L., "Projection Moiré Interferometry Measurements of Micro Air Vehicle Wings," SPIE International Symposium on Optical Science and Technology, San Diego, CA, August 4-6, 2001

${ }^{12}$ DeLuca, A., Reeder, M., Michael, V., Freeman, J., Bautista, I., Simonich, M., "Experimental Investigation into the Aerodynamics of a Flexible and Rigid Micro Air Vehicle," $24^{\text {th }}$ AIAA Aerodynamic Measurement Technology and Ground Testing Conference, Portland, OR, June 28-July 1, 2004

${ }^{13}$ Sutton, M., Cheng, M., Peters, W., Chao, Y., McNeill, S., "Application of an Optimized Digital Correlation Method to Planar Analysis," Image and Vision Computing, Vol. 4, pp 143-151, 1986

${ }^{14}$ Schreier, H., Braasch, J., Sutton, M., "Systematic Errors in Digital Image Correlation Caused by Gray-value Interpolation," Optical Engineering, Vol. 39, pp 2915-2921, 2000

${ }^{15}$ Thakur, S., Wright, J., Shyy, W., "STREAM: A Computational Fluid Dynamics and Heat Transfer Code for Complex Geometries. Part 1: Theory. Part 2: User's Guide," Department of Mechanical and Aerospace Engineering Technical Report, University of Florida, 2002

${ }^{16}$ Cook, R., Malkus, D., Plesha, M., Witt, R., “Concepts and Applications of Finite Element Analysis,” John Wiley and Sons, New York, 2002

${ }^{17}$ Jenkins, C., editor, "Gossamer Spacecraft: Membrane/Inflatable Structure Technology for Space Applications," AIAA Progress in Astronautics and Aeronautics Series, Vol. 191, 2001

${ }^{18}$ Systma, M., "Aerodynamic Flow Characterization of Micro Air Vehicles Utilizing Flow Visualization Methods," Masters Thesis, Department of Mechanical and Aerospace Engineering, Gainesville, FL, 2006 